\title{
Can the Discretization Modify the Performance of Schwarz Methods?
}

\author{
Victorita Dolean $^{1}$ and Martin J. Gander ${ }^{2}$ \\ 1 Univ. de Nice Sophia-Antipolis, Laboratoire J.-A. Dieudonné, UMR CNRS 6621, \\ Parc Valrose, 06108 Nice Cedex 02, France. dolean@unice.fr \\ 2 Section de Mathématiques, Université de Genève, CP 64, 1211 Genève 4, \\ martin.gander@unige.ch
}

Summary. Schwarz domain decomposition methods can be analyzed both at the continuous and discrete level. For consistent discretizations, one would naturally expect that the discretized method performs as predicted by the continuous analysis. We show in this short note for two model problems that this is not always the case, and that the discretization can both increase and decrease the convergence speed predicted by the continuous analysis.

\section{Introduction}

Classical Schwarz methods have been analyzed historically both at the continuous and the discrete level, see for example Schwarz [1870], Lions [1988, 1989, 1990], Quarteroni and Valli [1999] and references therein for continuous analysis, Dryja and Widlund [1987], Toselli and Widlund [2004] and references therein for analysis at the discrete level. Over the last decade, optimized Schwarz methods have been extensively developed at the continuous level. These methods converge significantly faster than the classical Schwarz methods, see for example Gander [2006], and references therein. More recently, Schwarz methods have also been developed for systems of partial differential equations, see for example Dolean et al. [2004] for Euler equations, Dolean and Gander [2008] for the Cauchy-Riemann equations, or Alonso Rodríguez and Gerardo-Giorda [2006], Dolean et al. [2009] for Maxwell's equations, and it was observed in two particular cases that a discretized Schwarz method converged faster than predicted by the continuous analysis. The purpose of this note is to explain this observation for the case of the Cauchy-Riemann equations, and also to reveal a previously not observed discrepancy for the case of the positive definite Helmholtz operator, $\eta-\Delta, \eta>0$. 


\section{The Cauchy-Riemann Equations}

Classical and optimized Schwarz methods have been analyzed in Dolean and Gander [2008] at the continuous level for the Cauchy Riemann equations,

$$
\mathcal{L} \mathbf{u}:=\sqrt{\eta} \mathbf{u}+A \partial_{x} \mathbf{u}+B \partial_{y} \mathbf{u}=\mathbf{f}, \quad A=\left[\begin{array}{cc}
-1 & 0 \\
0 & 1
\end{array}\right], \quad B=\left[\begin{array}{ll}
0 & 1 \\
1 & 0
\end{array}\right]
$$

and it was observed in the classical Schwarz case that the discretized algorithms converged faster than predicted by the continuous analysis. The finite volume discretization used in these experiments was on a Cartesian mesh with mesh points $x_{l m}=(l \Delta x, m \Delta y), l, m \in \mathbb{Z}$, namely

$$
\begin{gathered}
L \mathbf{u}_{l, m}:=\left(\begin{array}{c}
L_{1} \mathbf{u}_{l, m} \\
L_{2} \mathbf{u}_{l, m}
\end{array}\right)=\left(\begin{array}{c}
f_{l, m} \\
g_{l, m}
\end{array}\right)=: \mathbf{f}_{l, m}, \\
L_{1} \mathbf{u}_{l, m}:=\sqrt{\eta} u_{l, m}+\left(-D_{x}^{+}-\frac{D_{y}^{+}-D_{y}^{-}}{2}\right) u_{l, m}+\frac{D_{y}^{+}+D_{y}^{-}}{2} v_{l, m}, \\
L_{2} \mathbf{u}_{l, m}:=\sqrt{\eta} v_{l, m}+\left(D_{x}^{-}-\frac{D_{y}^{+}-D_{y}^{-}}{2}\right) v_{l, m}+\frac{D_{y}^{+}+D_{y}^{-}}{2} u_{l, m},
\end{gathered}
$$

where $D_{x}^{ \pm}$and $D_{y}^{ \pm}$are the usual finite difference operators in $x$ and $y$ directions. We consider now a decomposition of $\Omega=\mathbb{R}^{2}$ into two subdomains $\Omega_{1}=(-\infty, a) \times \mathbb{R}$ and $\Omega_{2}=(b, \infty) \times \mathbb{R}$. In the interior of $\Omega_{1}$ the equation (2) is verified for all $l<l_{1}$ and for $\Omega_{2}$, it is verified for all $l>l_{2}$. A discrete Schwarz algorithm with general transmission conditions is

$$
\begin{array}{rlrl}
L \mathbf{u}_{l, m}^{1, n} & =\mathbf{f}_{l, m}, \quad l<l_{1}, & L \mathbf{u}_{l, m}^{2, n} & =\mathbf{f}_{l, m}, \quad l>l_{2}, \\
L_{2} \mathbf{u}_{l_{1}, m} & =g_{l_{1}, m}, & L_{1} \mathbf{u}_{l_{2}, m} & =f_{l_{2}, m}, \\
u_{l_{1}, m}^{1, n}+S^{1} v_{l_{1}, m}^{1, n} & =u_{l_{1}, m}^{2, n-1}+S^{1} v_{l_{1}, m}^{2, n-1}, v_{l_{2}, m}^{2, n}+S^{2} u_{l_{2}, m}^{2, n} & =v_{l_{2}, m}^{1, n-1}+S^{2} u_{l_{2}, m}^{1, n} .
\end{array}
$$

where $l_{1}, l_{2}$ are the indices of the interface points, and $S^{1,2}$ are finite difference operators that may contain parameters chosen in order to obtain better convergence than with the classical algorithm. If only information following the characteristics are exchanged, $S^{1,2} \equiv 0$, we obtain the classical Schwarz algorithm, see Dolean and Gander [2008].

To simplify the analysis, we use the same discretization step in the $x$ and $y$ direction, $h:=\Delta x=\Delta y$. We denote the overlap parameter by $\delta:=l_{1}-l_{2}$, and use a discrete Fourier transform to study convergence properties of algorithm (3). Since we study the evolution of the error, it is sufficient to study the homogeneous counterpart of (3), and we look for the solutions of the form

$$
\mathbf{u}_{l, m}^{j, n}=\sum_{k} \alpha^{j, n}(k) e^{l h \lambda(k)} e^{i k m h}\left(\begin{array}{c}
\hat{u}_{k, m}^{j, n} \\
\hat{v}_{k, m}^{j, n}
\end{array}\right)
$$

where $j=1,2$ denotes the subdomain index and $n$ the iteration number of the Schwarz algorithm. At each iteration and in each subdomain, the iterates satisfy for each discrete frequency $k$ the system of equations 


$$
\begin{aligned}
& \sqrt{\eta} \hat{u}_{k, m}-\frac{e^{\lambda(k) h}-1}{h} \hat{u}_{k, m}+\frac{2-e^{i k h}-e^{-i k h}}{2 h} \hat{u}_{k, m}+\frac{e^{i k h}-e^{-i k h}}{2 h} \hat{v}_{k, m}=0, \\
& \sqrt{\eta} \hat{v}_{k, m}+\frac{1-e^{-\lambda(k) h}}{h} \hat{v}_{k, m}+\frac{2-e^{i k h}-e^{-i k h}}{2 h} \hat{v}_{k, m}+\frac{e^{i k h}-e^{-i k h}}{2 h} \hat{u}_{k, m}=0 .
\end{aligned}
$$

If we denote by $\phi:=\frac{e^{\lambda(k) h}-1}{h}$ and by $\hat{w}:=\frac{\hat{v}_{k, m}}{\hat{u}_{k, m}}$, we obtain from the first equation of (5) that

$$
\phi=\sqrt{\eta}+\frac{a_{k} h}{2}+b_{k} \hat{w}
$$

where $b_{k}=\frac{i \sin (k h)}{h}=i k+\mathcal{O}(h)$ and $a_{k}=\frac{2(1-\cos (k h))}{h^{2}}=k^{2}+\mathcal{O}(h)$ are the symbols of the discrete first and second order derivative with respect to $y$. Replacing this result into the second equation of (5) we obtain an equation for $\hat{w}$,

$$
\begin{aligned}
b_{k}\left(\left(\sqrt{\eta}+\frac{a_{k} h}{2}\right) h+1\right) \hat{w}^{2} & +\left(\left(\sqrt{\eta}+\frac{a_{k} h}{2}\right)\left(\left(\sqrt{\eta}+\frac{a_{k} h}{2}\right) h+2\right)+b_{k}^{2} h\right) \hat{w} \\
+ & b_{k}\left(\left(\sqrt{\eta}+\frac{a_{k} h}{2}\right) h+1\right)=0 .
\end{aligned}
$$

This equation has solutions $\hat{w}_{1,2}$, which give two corresponding values of $\phi_{1,2}$ with opposite signs, whose asymptotic behavior for $h$ small is

$$
\phi_{1,2}(k, h)= \pm \sqrt{\eta+k^{2}}+\mathcal{O}(h) .
$$

Since subdomain solutions need to remain bounded, they must be of the form

$$
\mathbf{u}_{l, m}^{j, n}=\sum_{k} \alpha^{j, n}(k)\left(\phi_{j} h+1\right)^{l} e^{i k m h}\left(\begin{array}{c}
\hat{u}_{k, m}^{j, n} \\
\hat{v}_{k, m}^{j, n}
\end{array}\right) .
$$

If we denote by $\sigma_{1,2}$ the Fourier symbols of the operators $S^{1,2}$, and insert (7) into the interface conditions of algorithm (3), we obtain for each frequency $k$

$$
\begin{aligned}
& \alpha^{1, n}(k)\left(\hat{u}_{k, m}^{1}+\sigma_{1} \hat{v}_{k, m}^{1}\right)\left(\phi_{1} h+1\right)^{l_{1}}=\alpha^{2, n-1}(k)\left(\hat{u}_{k, m}^{2}+\sigma_{1} \hat{v}_{k, m}^{2}\right)\left(\phi_{2} h+1\right)^{l_{1}}, \\
& \alpha^{2, n}(k)\left(\hat{v}_{k, m}^{2}+\sigma_{2} \hat{u}_{k, m}^{2}\right)\left(\phi_{2} h+1\right)^{l_{2}}=\alpha^{1, n-1}(k)\left(\hat{v}_{k, m}^{1}+\sigma_{2} \hat{u}_{k, m}^{1}\right)\left(\phi_{1} h+1\right)^{l_{2}} .
\end{aligned}
$$

Taking into account that $\hat{w}=\frac{\hat{v}_{k, m}}{\hat{u}_{k, m}}$ and using (6), the convergence factor of algorithm (3) is

$$
\begin{aligned}
& \rho(k, \eta, \delta, h)=\left(\frac{\alpha^{2, n}}{\alpha^{2, n-2}}\right)^{\frac{1}{2}}=\left(\frac{1+\sigma_{1} \hat{v}_{2}}{1+\sigma_{1} \hat{v}_{1}} \cdot \frac{\sigma_{2}+\hat{v}_{1}}{\sigma_{2}+\hat{v}_{2}}\right)^{\frac{1}{2}} \cdot\left(\frac{\phi_{2} h+1}{\phi_{1} h+1}\right)^{\frac{\delta}{2}} \\
& \quad=\left(\frac{b_{k}+\sigma_{1}\left(\phi_{2}-\sqrt{\eta}-\frac{a_{k} h}{2}\right)}{b_{k}+\sigma_{1}\left(\phi_{1}-\sqrt{\eta}-\frac{a_{k} h}{2}\right)} \cdot \frac{b_{k} \sigma_{2}+\left(\phi_{1}-\sqrt{\eta}-\frac{a_{k} h}{2}\right)}{b_{k} \sigma_{2}+\left(\phi_{2}-\sqrt{\eta}-\frac{a_{k} h}{2}\right)}\right)^{\frac{1}{2}} \cdot\left(\frac{\phi_{2} h+1}{\phi_{1} h+1}\right)^{\frac{\delta}{2}} .
\end{aligned}
$$

The maximum $\rho_{\max }$ of this convergence factor over all relevant frequencies $k \in\left[0, k_{\max }\right]$, with the estimate $k_{\max }=\frac{\pi}{h}$, determines the overall contraction factor of the method, and hence the rate of convergence of the associated algorithm. Different classes of interface conditions were studied at the continuous level in Dolean and Gander [2008]:

Case 1: $\sigma_{1}=\sigma_{2}=0$. This case corresponds to the classical Schwarz algorithm which exchanges characteristic information at the interfaces. 
Proposition 1. Let $\sigma_{1}=\sigma_{2}=0$. In the non-overlapping case of algorithm (3) , $\delta=0$, the convergence factor attains its maximum for $h$ small at $k_{b}=$ $2^{\frac{1}{2}} \cdot 3^{-\frac{1}{4}} \eta^{\frac{1}{8}} \cdot h^{-\frac{3}{4}}$, which leads to the overall contraction factor

$$
\rho_{\text {max }}:=\rho\left(k_{b}, \eta, 0, h\right)=1-2^{\frac{3}{2}} \cdot 3^{-\frac{3}{4}} \eta^{\frac{3}{8}} h^{\frac{3}{4}}+\mathcal{O}(h) .
$$

In the overlapping case of algorithm (3), we have for

$$
\begin{array}{lll}
\delta=1: & k_{b}=\eta^{\frac{1}{4}} \cdot h^{-\frac{1}{2}}, & \rho_{\max }=1-2 \eta^{\frac{1}{4}} \cdot h^{\frac{1}{2}}+\mathcal{O}(h), \\
\delta=2: & k_{b}=\eta^{\frac{1}{4}} \cdot 2^{-\frac{1}{2}} \cdot h^{-\frac{1}{2}}, & \rho_{\max }=1-2^{\frac{3}{2}} \eta^{\frac{1}{4}} \cdot h^{\frac{1}{2}}+\mathcal{O}(h) .
\end{array}
$$

Remark 1. In the non-overlapping case, the convergence factor predicted by the continuous analysis in Dolean and Gander [2008] was $1-\mathcal{O}(h)$, but faster convergence was observed numerically, a gap closed by the present analysis. In the overlapping case however, for $\delta=1,2$ and probably also bigger $\delta$, the convergence factors from the discrete and continuous analysis have the same asymptotic behavior, see Dolean and Gander [2008].

Case 2: $\sigma_{1}=\frac{b_{k}}{\sqrt{\eta}+p}, \sigma_{2}=\frac{\sqrt{\eta}-p}{b_{k}}$, a case with one parameter $p>0$ to choose for best performance. Since $b_{k}$ is the discrete symbol of the tangential derivative, this case corresponds to the optimized algorithm where local operators are used in the transmission conditions expressed with first order derivatives. Note that even if we have $b_{k}$ in the denominator, it suffices to multiply both sides of the transmission conditions with $b_{k}$ in order to obtain local operators.

Proposition 2. Let $\sigma_{1}=\frac{b_{k}}{\sqrt{\eta}+p}$ and $\sigma_{2}=\frac{\sqrt{\eta}-p}{b_{k}}$. In the non-overlapping case of algorithm (3), $\delta=0$, the optimized parameter $p^{*}$ is for $h$ small solution of

$$
\rho\left(k_{1}(p), \eta, 0, h, p\right)=\rho\left(k_{\max }, \eta, 0, h, p\right),
$$

where $k_{1}(p)$ is a maximum of $\rho$, and we have the asymptotic result

$$
k_{1}=\frac{C_{k_{1}}}{h}, \quad p^{*}=\frac{C p}{\sqrt{h}}, \quad \rho_{\max }=1-\frac{1}{4 C_{p}} \cdot\left(3 C_{p}^{2}+8 \sqrt{\eta}\right) \sqrt{h}+\mathcal{O}(h) .
$$

The constants $C_{k_{1}}$ and $C_{p}$ can be explicitly computed: if $\theta$ denotes the real root of $6 x^{3}-20 x^{2}+19 x-3=0$, then we get $C_{k_{1}}=\arccos (\theta)=1.373593$, and

$$
\begin{aligned}
C p= & 2 /\left(-3 \cdot \cos \left(C_{k_{1}}\right)^{3}+6 \cdot \cos \left(C_{k_{1}}\right)^{2}+3 \cdot \cos \left(C_{k_{1}}\right)-6\right. \\
& \left.+16 \cdot\left(\left(-1+\cos \left(C_{k_{1}}\right)\right) \cdot\left(3 \cdot \cos \left(C_{k_{1}}\right)-5\right)\right)^{1 / 2}\right) \cdot\left(-\left(6 \cdot \cos \left(C_{k_{1}}\right)^{3}\right.\right. \\
& -12 \cdot \cos \left(C_{k_{1}}\right)^{2}-6 \cdot \cos \left(C_{k_{1}}\right)+12 \\
& \left.-32 \cdot\left(\left(-1+\cos \left(C_{k_{1}}\right)\right) \cdot\left(3 \cdot \cos \left(C_{k_{1}}\right)-5\right)\right)^{1 / 2}\right) \cdot \eta^{1 / 2} \cdot\left(\cos \left(C_{k_{1}}\right)^{3}\right. \\
& \left.\left.-2 \cdot \cos \left(C_{k_{1}}\right)^{2}-\cos \left(C_{k_{1}}\right)+2\right)\right)^{1 / 2} \\
= & 0.7460898 \cdot \eta^{\frac{1}{4}} .
\end{aligned}
$$


Proposition 3. Let $\sigma_{1}=\frac{b_{k}}{\sqrt{\eta}+p}$ and $\sigma_{2}=\frac{\sqrt{\eta}-p}{b_{k}}$. In the overlapping case, $\delta=1$, the optimized parameter $p^{*}$ is for $h$ small solution of the equation

$$
\rho\left(k_{1}(p), \eta, \delta, h, p\right)=\rho\left(k_{\min }, \eta, \delta, h, p\right),
$$

where again $k_{1}(p)$ is a maximum of $\rho$, and $k_{\min } \geq 0$ is the minimum frequency on the interface, and we have asymptotically

$$
\begin{gathered}
p^{*}=2^{-\frac{1}{3}} \cdot\left(k_{\min }^{2}+\eta\right)^{\frac{1}{3}} \cdot h^{-\frac{1}{3}}, \quad k_{1}=2^{\frac{1}{3}} \cdot\left(k_{\min }^{2}+\eta\right)^{\frac{1}{6}} \cdot h^{-\frac{2}{3}}, \\
\rho_{\max }=1-4 \cdot\left(\eta+k_{\min }^{2}\right)^{\frac{1}{6}} \cdot 2^{\frac{1}{3}} \cdot h^{\frac{1}{3}}+\mathcal{O}(h) .
\end{gathered}
$$

The same asymptotic behavior is also obtained for bigger overlap, $\delta>1$.

Remark 2. In both Propositions 2 and 3, the asymptotic analysis of the discretized algorithm presented here and the continuous algorithm from Dolean and Gander [2008] predict the same asymptotic performance.

Case 3: $\sigma_{1}=\sigma_{2}=\sigma=\frac{b_{k}}{\sqrt{\eta}+p}$, where we can again choose $p>0$ for best performance.

Proposition 4. The optimized parameter $p^{*}$ is for $h$ small solution of the equation

$$
\rho\left(k_{1}(p), \eta, \delta, h, p\right)=\rho\left(k_{2}(p), \eta, \delta, h, p\right),
$$

where $k_{1}(p)$ and $k_{2}(p)$ are maxima of $\rho$. In the case $\delta \leq 3$, which means no or small overlap (at most 3 mesh cells), we have the asymptotic result

$$
\begin{gathered}
p^{*}=\frac{C p}{h}, \quad k_{1}=\frac{C_{k_{1}}}{\sqrt{h}}, \quad k_{2}=\frac{C_{k_{2}}}{h}, \\
\rho_{\max }=1-2 \frac{2 C_{k_{1}}^{2}+C_{p} \sqrt{\eta}+\delta C_{k_{1}}^{2} C_{p}}{C_{p} C_{k_{1}}} \sqrt{h}+\mathcal{O}(h) .
\end{gathered}
$$

In the case with more overlap, $\delta \geq 4$, we obtain for $h$ small

$$
\begin{gathered}
p^{*}=\frac{C p}{\sqrt{h}}, \quad k_{1}=\frac{C_{k_{1}}}{h^{\frac{1}{4}}}, \quad k_{2}=\frac{C_{k_{2}}}{h^{\frac{3}{4}}}, \\
\rho_{\max }=1-2 \frac{2 C_{k_{1}}^{2}+C_{p} \sqrt{\eta}}{C_{p} C_{k_{1}}} h^{\frac{1}{4}}+\mathcal{O}\left(h^{\frac{1}{2}}\right) .
\end{gathered}
$$

The constants can again be computed: for example for the zero or small overlapping case, we obtain

$$
\begin{aligned}
& \delta=0: \quad C_{p}=0.383205, C_{k_{1}}=0.437724 \eta^{\frac{1}{4}}, C_{k_{2}}=2.29295, \\
& \delta=1: \quad C_{p}=0.068781, C_{k_{1}}=0.182338 \eta^{\frac{1}{4}}, C_{k_{2}}=2.71717,
\end{aligned}
$$

and for a case with bigger overlap, $\delta=4$, we get

$$
C_{p}=\frac{1}{2} \eta^{\frac{1}{4}}, C_{k_{1}}=\frac{1}{2} \eta^{\frac{3}{8}}, C_{k_{2}}=\frac{1}{2} \eta^{\frac{1}{8}} .
$$

We observed that for $\delta>4$ the factor one half in the constants (14) is replaced by a factor that becomes smaller and smaller, as $\delta$ becomes larger. 
Remark 3. Again there is a substantial difference between the continuous analysis from Dolean and Gander [2008] and the discrete analysis presented here: the continuous analysis predicted the convergence factor $1-\mathcal{O}\left(h^{\frac{1}{3}}\right)$ without overlap, and $1-\mathcal{O}\left(h^{\frac{1}{4}}\right)$ with overlap.

Such differences are not only restricted to the somewhat exotic example of the Cauchy-Riemann equations, they were also observed when the classical Schwarz method is applied to Maxwell's equations, see Dolean et al. [2009], and we will show in the next section that even in the case of simple positive definite scalar partial differential equations such differences can occur.

\section{The Positive Definite Helmholtz Equation}

Optimized Schwarz methods have been analyzed thoroughly for the positive definite Helmholtz equation at the continuous level in Gander [2006], and extensive numerical tests have been presented which illustrate the performance predicted by the continuous analysis. We show in this section that there are certain, quite natural discretizations which can lead again to differences in the performance.

We use the same Cartesian mesh on $\Omega=\mathbb{R}^{2}$ with mesh points $x_{l, m}=$ $(l \Delta x, m \Delta y), l, m \in \mathbb{Z}$, and we consider the five point finite difference discretization of the positive definite Helmholtz equation $(\eta-\Delta) u=f$,

$$
L u_{l, m}:=\left(\eta-D_{x}^{+} D_{x}^{-}-D_{y}^{+} D_{y}^{-}\right) u_{l, m}=f_{l, m} .
$$

With the same decomposition as in section 2, a general discrete Schwarz algorithm applied to (15) is

$$
\begin{array}{rlrlrl}
L u_{l, m}^{1, n} & =f_{l_{, m},}, & l<l_{1}, & L u_{l, m}^{2, n} & =f_{l, m}, & l>l_{2}, \\
B_{1} u_{l_{1}, m}^{1, n} & =B_{1} u_{l_{1}, m}^{2, n-1}, & j \in \mathbb{Z}, & B_{2} u_{l_{2}, m}^{2, n}=B_{2} u_{l_{2}, m}^{1, n-1}, & j \in \mathbb{Z},
\end{array}
$$

where $B_{1,2}$ denote the discrete transmission conditions (Dirichlet or Robin). We set again $h:=\Delta x=\Delta y$ and $\delta:=l_{1}-l_{2}$ for the overlap. Using a discrete Fourier analysis in the $y$ direction, one can show the following results:

Proposition 5. For Dirichlet transmission conditions, $B_{1,2}=I d$, and one mesh size overlap, $\delta=1$, the asymptotic convergence factor of algorithm (16) for $h$ small is given by

$$
\rho_{\max }=1-\sqrt{\eta} h+\mathcal{O}\left(h^{2}\right),
$$

which is identical to the result obtained from a continuous analysis.

Proposition 6. For Robin transmission conditions discretized by one-sided finite differences, $B_{1}:=D_{x}^{-}+p$ and $B_{2}:=D_{x}^{+}-p$, the optimized Robin 
parameter and asymptotic convergence factor of algorithm (16) for $h$ small are given by

$$
\begin{array}{lll}
\delta=1: & p^{*}=2^{\frac{1}{4}} \eta^{\frac{1}{4}} h^{-\frac{1}{2}}, \quad \rho_{\max }=1-\eta^{\frac{1}{4}} 2^{\frac{3}{4}} \sqrt{h}+\mathcal{O}(h), \\
\delta=2: & p^{*}=2^{-\frac{1}{3}} \eta^{\frac{1}{3}} h^{-\frac{1}{3}}, & \rho_{\max }=1-2 \eta^{\frac{1}{6}} 2^{\frac{1}{3}} h^{\frac{1}{3}}+\mathcal{O}\left(h^{\frac{2}{3}}\right) .
\end{array}
$$

Remark 4. In the case $\delta=2$ with overlap $2 h$, and one-sided finite difference discretization of the normal derivative, the asymptotic performance of the discretized algorithm is as predicted by the continuous analysis, see Gander [2006]. However with $\delta=1$, which means minimal overlap, the asymptotic performance of the discretized overlapping algorithm is worse, like predicted for the non-overlapping algorithm by the continuous analysis in Gander [2006]. The benefit of the overlap is thus lost with this discretization!

For Robin transmission conditions obtained by centered finite differences, the algorithm (16) is given by

$$
\begin{aligned}
L u_{l, m}^{1, n} & =f_{l, m}, \quad l<l_{1}, j \in \mathbb{Z} \\
\left(D_{x}^{-}+\left(\eta-D_{y}^{+} D_{y}^{-}\right) \frac{h}{2}+p\right) u_{l_{1, m}, m}^{1, n} & =\left(D_{x}^{+}-\left(\eta-D_{y}^{+} D_{y}^{-}\right) \frac{h}{2}+p\right) u_{l_{1}, m}^{2, n-1}+h f_{l_{1}, m} \\
L u_{l, m}^{2, n} & =f_{l, m}, \quad l>l_{2}, j \in \mathbb{Z} \\
\left(D_{x}^{+}-\left(\eta-D_{y}^{+} D_{y}^{-}\right) \frac{h}{2}-p\right) u_{l_{2}, m}^{2, n} & =\left(D_{x}^{-}+\left(\eta-D_{y}^{+} D_{y}^{-}\right) \frac{h}{2}-p\right) u_{l_{2}, m}^{1, n-1}-h f_{l_{2}, m}
\end{aligned}
$$

Proposition 7. For the discrete optimized Schwarz algorithm (18), the optimized Robin parameter and asymptotic convergence factor are for $h$ small given by

$$
\begin{array}{ll}
\delta=0: p^{*}=2^{\frac{1}{4}} \sqrt{\frac{2^{\frac{1}{2}}+1}{4+3 \cdot 2^{\frac{1}{2}}}} \eta^{\frac{1}{4}} h^{-\frac{1}{2}}, & \rho_{\max }=1-2 \eta^{\frac{1}{4}}\left(2+3 \cdot 2^{-\frac{1}{2}}\right)^{\frac{1}{2}} \sqrt{h}+\mathcal{O}(h), \\
\delta=1: p^{*}=2^{-\frac{1}{3}} \eta^{\frac{1}{3}} h^{-\frac{1}{3}}, & \rho_{\max }=1-4 \eta^{\frac{1}{6}} 2^{\frac{1}{3}} h^{\frac{1}{3}}+\mathcal{O}\left(h^{\frac{2}{3}}\right) .
\end{array}
$$

Remark 5. With the centered finite difference approximation of the normal derivative, the discretized optimized Schwarz algorithm for the positive definite Helmholtz equation has the same asymptotic convergence behavior as predicted by the continuous analysis in Gander [2006].

\section{Conclusions}

As we have seen, the discretization can modify the convergence behavior of Schwarz algorithms, compared to the predicted behavior by a continuous analysis. We note however that in all cases we have analyzed, different behavior is only observed when the overlap is sufficiently small. In the case of enough overlap, the results of the discrete and continuous analysis are consistent. This observation suggests that the reason for possibly different behavior of the discrete algorithm could lie in the fact that the physical properties are in those cases not well enough resolved in the overlapping region of very few grid points. 


\section{References}

Ana Alonso Rodríguez and Luca Gerardo-Giorda. New nonoverlapping domain decomposition methods for the harmonic Maxwell system. SIAM J. Sci. Comput., 28(1):102-122 (electronic), 2006. ISSN 1064-8275.

Victorita Dolean and Martin J. Gander. Why classical Schwarz methods applied to certain hyperbolic systems converge even without overlap. In Domain decomposition methods in science and engineering XVII, volume 60 of Lect. Notes Comput. Sci. Eng., pages 467-475. Springer, Berlin, 2008.

Victorita Dolean, Martin J. Gander, and Luca Gerardo-Giorda. Optimized schwarz methods for Maxwell's equations. SIAM J. Sci. Comput., 31(3): 2193-2213, 2009.

Victorita Dolean, Stephane Lanteri, and Frederic Nataf. Convergence analysis of a Schwarz type domain decomposition method for the solution of the Euler equations. Appl. Num. Math., 49:153-186, 2004.

Maksymilian Dryja and Olof B. Widlund. An additive variant of the Schwarz alternating method for the case of many subregions. Technical Report 339, also Ultracomputer Note 131, Department of Computer Science, Courant Institute, 1987.

Martin J. Gander. Optimized Schwarz methods. SIAM J. Numer. Anal., 44 (2):699-731 (electronic), 2006. ISSN 0036-1429.

Pierre-Louis Lions. On the Schwarz alternating method. I. In Roland Glowinski, Gene H. Golub, Gérard A. Meurant, and Jacques Périaux, editors, First International Symposium on Domain Decomposition Methods for Partial Differential Equations, pages 1-42, Philadelphia, PA, 1988. SIAM.

Pierre-Louis Lions. On the Schwarz alternating method. II. In Tony Chan, Roland Glowinski, Jacques Périaux, and Olof Widlund, editors, Domain Decomposition Methods, pages 47-70, Philadelphia, PA, 1989. SIAM.

Pierre-Louis Lions. On the Schwarz alternating method. III: a variant for nonoverlapping subdomains. In Tony F. Chan, Roland Glowinski, Jacques Périaux, and Olof Widlund, editors, Third International Symposium on Domain Decomposition Methods for Partial Differential Equations, held in Houston, Texas, March 20-22, 1989, Philadelphia, PA, 1990. SIAM.

Alfio Quarteroni and Alberto Valli. Domain Decomposition Methods for Partial Differential Equations. Oxford Science Publications, 1999.

H. A. Schwarz. Über einen Grenzübergang durch alternierendes Verfahren. Vierteljahrsschrift der Naturforschenden Gesellschaft in Zürich, 15:272286, May 1870.

Andrea Toselli and Olof Widlund. Domain Decomposition Methods - Algorithms and Theory, volume 34 of Springer Series in Computational Mathematics. Springer, 2004. 